\title{
Closing in on HED meteorite sources
}

\author{
Mark V. Sykes ${ }^{1}$ and Faith Vilas ${ }^{2}$ \\ ${ }^{1}$ Steward Observatory, University of Arizona, Tucson, Arizona 85721, U.S.A. \\ ${ }^{2}$ NASA Johnson Space Center, SN3, Houston, Texas 77058, U.S.A.
}

(Received March 25, 2001; Revised June 1, 2001; Accepted June 28, 2001)

\begin{abstract}
Members of the Vesta dynamical family have orbital elements consistent with ejecta from a single large excavating collision from a single hemisphere of Vesta. The portion of Vesta's orbit at which such an event must have occurred is slightly constrained and depends on the hemisphere impacted. There is some evidence for subsequent disruptions of these ejected objects. Spectroscopy suggests that the dynamical family members are associated with material from Vesta's interior that was excavated by the event giving rise to the crater covering much of Vesta's southern hemisphere. The 505-nm pyroxene feature seen in HED meteorites is relatively absent in a sample of Vesta dynamical family members raising the question of whether this collisional event was the source of the HED meteorites. Asteroids spectroscopically associated with Vesta that possess this feature are dynamically distinct and could have arisen from a different collisional event on Vesta or originated from a body geochemically similar to Vesta that was disrupted early in the history of the solar system.
\end{abstract}

\section{Introduction}

The howardite-eucrite-diogenite (HED) meteorites comprise $\sim 6 \%$ of present meteorite falls (McSween, 1999). The chemical and mineralogical compositions of these meteorites have been analyzed, and the spectral reflectance characteristics have been measured (cf., Hiroi et al., 1994; Gaffey, 1997). Similarity between the depth, width, and spectral placement of the 1- and 2- $\mu$ m mafic silicate absorption features in the reflectance spectra of these HED meteorites and the asteroid 4 Vesta has been used to argue that Vesta is at the least an analogue for the HED meteorites (Consolmagno and Drake, 1977; Drake, 1979) or their parent body.

During the 1980s, theoretical studies showed that the dynamical transfer of objects to Earth orbit was possible from the 3:1 Kirkwood gap located between 2.45 and 2.55 AU, and the $v_{6}$ resonance which defines the inner edge of the main asteroid belt located near $2 \mathrm{AU}$ at low inclinations (Wisdom, 1987; Wetherill, 1988). With a mean semimajor axis of $2.36 \mathrm{AU}$, Vesta is located between, but not near, these two locations. The mystery of how putative pieces of Vesta were transported to the Earth remained unexplained until Binzel and Xu (1993) identified 20 asteroids near Vesta that showed the deep mafic silicate absorption feature near 1 micron (suggesting a spectrally-dominant pyroxene composition with ferrous iron) characteristic of Vesta's spectrum. These 'Vestoids' stretched in semimajor axis to form a bridge connecting $v_{6}$, Vesta, and the 3:1 Kirkwood gap and were hypothesized to be collisional fragments of Vesta.

Vesta is also known to have a dynamical family of asteroids associated with it (Zappala et al., 1995). Since the

Copy right(c) The Society of Geomagnetism and Earth, Planetary and Space Sciences (SGEPSS); The Seismological Society of Japan; The Volcanological Society of Japan; The Geodetic Society of Japan; The Japanese Society for Planetary Sciences. discovery of asteroid families by Hirayama (1918), such dynamical clusters of objects have been thought to arise from the collisional fragmentation and ejection of material from a parent object - usually associated with the largest family member (that traditionally provides the name for the family). Zappala et al. identified these objects on the basis of methods of determining dynamical proximity, defining a cluster. They define several levels of association arising from their analysis. Class 3 indicates the most reliable "family" status, while Class 1 indicates a lower reliable "cluster" member. Class 2 is intermediate. The orbital element distribution for 149 Class 3 Vesta dynamical family members and 38 Vestoids (identified in Binzel and Xu, 1993 and Bus, 1999) having known proper elements are shown in Fig. 1. Proper elements used in this paper are obtained from Knežević and Milani (2000).

During the 1994 and 1996 oppositions of Vesta, Hubble Space Telescope images taken that covered all of Vesta's surface, showing albedo differences many of which correspond to topographical differences. An impact crater measuring $460-\mathrm{km}$ diameter is located at Vesta's south pole (Thomas et al., 1997). Smaller impact craters are located near Vesta's equator (Thomas et al., 1997).

\section{The Origin of the Vesta Dynamical Family}

As has been pointed out by others (cf., Marzari et al., 1996), the proper inclinations of Class 3 Vesta dynamical family members tend to be larger than that of Vesta. This suggests that many of these dynamical family members were ejected preferentially from a single hemisphere. Since impactor arrival directions are expected to be isotropic relative to Vesta, it would appear that most of these ejected fragments arose from a singular collisional event (and that most, though not necessarily all, of the Class 3 dynamical family members had a common origin). Given the large crater cov- 


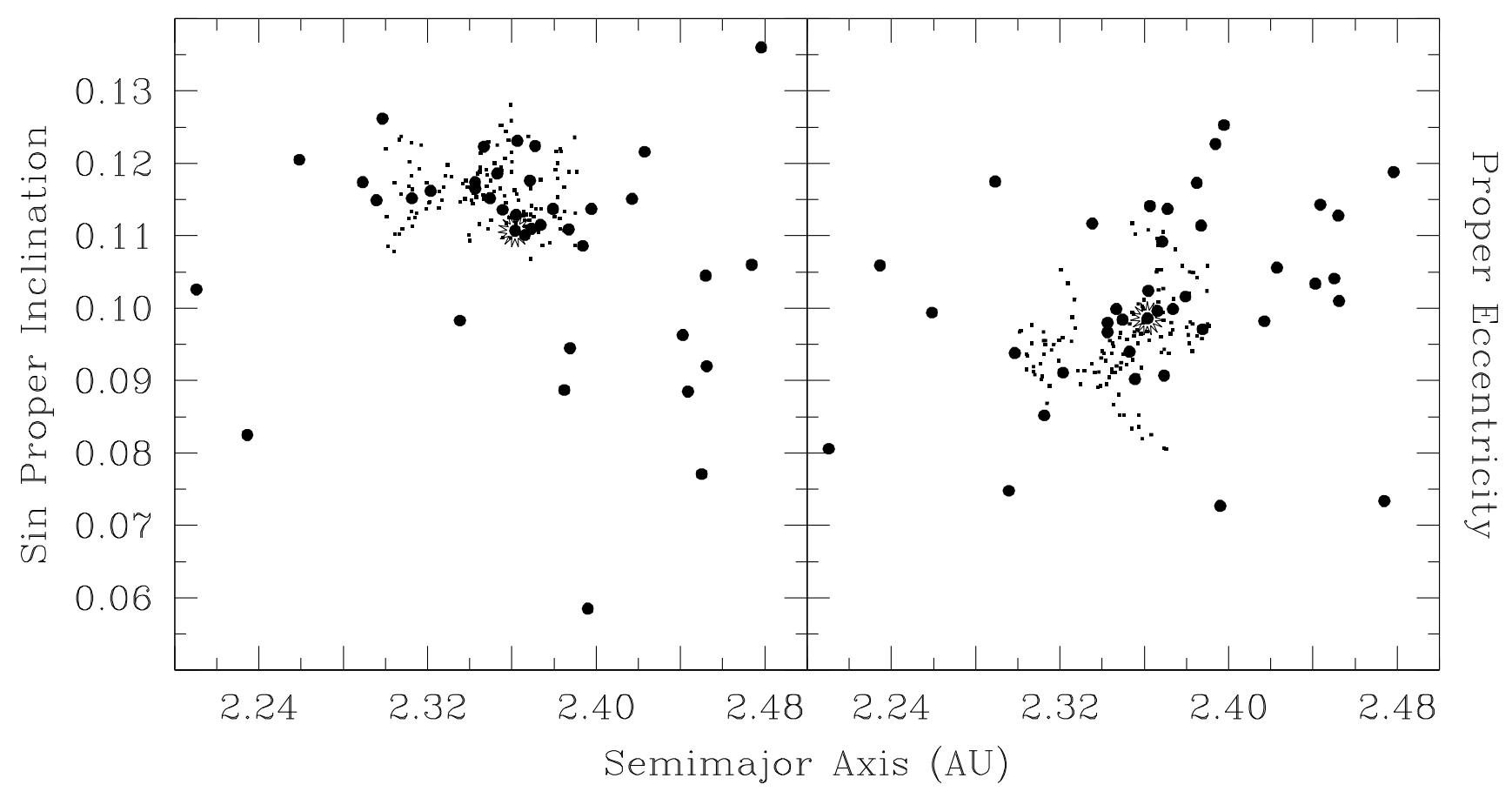

Fig. 1. Proper orbital elements of Class 3 Vesta family members (small dots) and Vestoids (large filled circles). Vesta is designated as a starburst.

ering Vesta's southern hemisphere, one is inclined to correlate the two. In Fig. 2 we compare the orbital elements of Class 3 dynamical family members to those of objects ejected with a velocity relative to Vesta's of $200 \mathrm{~m} / \mathrm{s}$ emitted uniformly from the (a) Northern and (b) Southern hemispheres over different ranges of mean anomaly of Vesta's present orbit. The northern impact was chosen for purposes of comparison. In Fig. 3 the same comparison is made for eccentricities. Simple visual inspection suggests that southern hemisphere ejection on the outbound portion of Vesta's orbit, rounding aphelion, would account for the dynamical family's inclinations, and northern hemisphere ejection on the inbound leg could also do the job. The eccentricity distributions suggest ejections occurred preferentially somewhere on the perihelion side of the orbit. Given that such an event occurred early in solar system history, at which time Vesta's orbit angles were different from today's values, the inclinations are probably not very constraining of the actual orbital location at which a family forming collisional event took place, though it is certainly indicative of hemispheric ejection. However, the eccentricity distribution does seem to retain a weak record that the event took place nearer perihelion than aphelion. In addition, the impact could have caused a shift in the orientation of Vesta, in which case a northern impact crater could have rotated to the southern location observed today.

We note some interesting structures within the eccentricity-semimajor axis distribution of the dynamical family members that seems to relate to the somewhat linear distributions seen near perihelion and aphelion model ejections. These structures suggest that the central concentration of family members derived from a Vesta event, while the outlying "orthogonal" structures occurred as a consequence of the subsequent disruptions of Vesta fragments. Thus the Vesta dynamical family may have experienced a complex collisional history.

\section{The Origin of Vestoids}

The Vestoids range in diameter from 3.9 to $10.3 \mathrm{~km}$ (assuming geometric albedos of 0.36). They cover a broader range of orbital element phase space than do the Class 3 Vesta dynamical family members (Fig. 1). If the latter were generated by a single impact event, it does not seem likely that some of the Vestoids shared the same origin with them - in particular, those Vestoids having semimajor axes $>2.44 \mathrm{AU}$ and lower proper inclinations than Vesta - unless they represent some antipodal spall. To accommodate the theory of common Vesta origin for both sets of objects, more than one collisional event on Vesta has been invoked (cf., Marzari et al., 1996).

If all of the Vestoids were ejected directly from the surface of Vesta, it is useful to look at the minimum relative velocities ( $V_{\text {rel }}$, of these asteroids compared to Vesta) by considering the envelope within orbital element phase space filled by test particles ejected at random directions from Vesta's surface at random locations in Vesta's orbit. The result is also shown in Fig. 4. The bulk of the Vestoids fall within the 400 $\mathrm{m} / \mathrm{s}$ envelope. To get all the outliers, however, one needs final relative velocities between 600 and $800 \mathrm{~m} / \mathrm{s}$. The velocity corresponding to ejection is higher than this, because one needs to include Vesta's escape velocity. Using Vesta's mass (Hilton, 1997) and mean radius (Thomas et al., 1997), the escape velocity from Vesta's mean surface is $394 \mathrm{~m} / \mathrm{s}$. The ejection velocity of these Vestoids from Vesta's surface would then be

$$
V_{\mathrm{ej}}=\left[V_{\mathrm{rel}}^{2}+V_{\mathrm{esc}}^{2}\right]^{1 / 2} .
$$

Thus, observed relative velocities of 200, 400, 600, and 800 
(a)

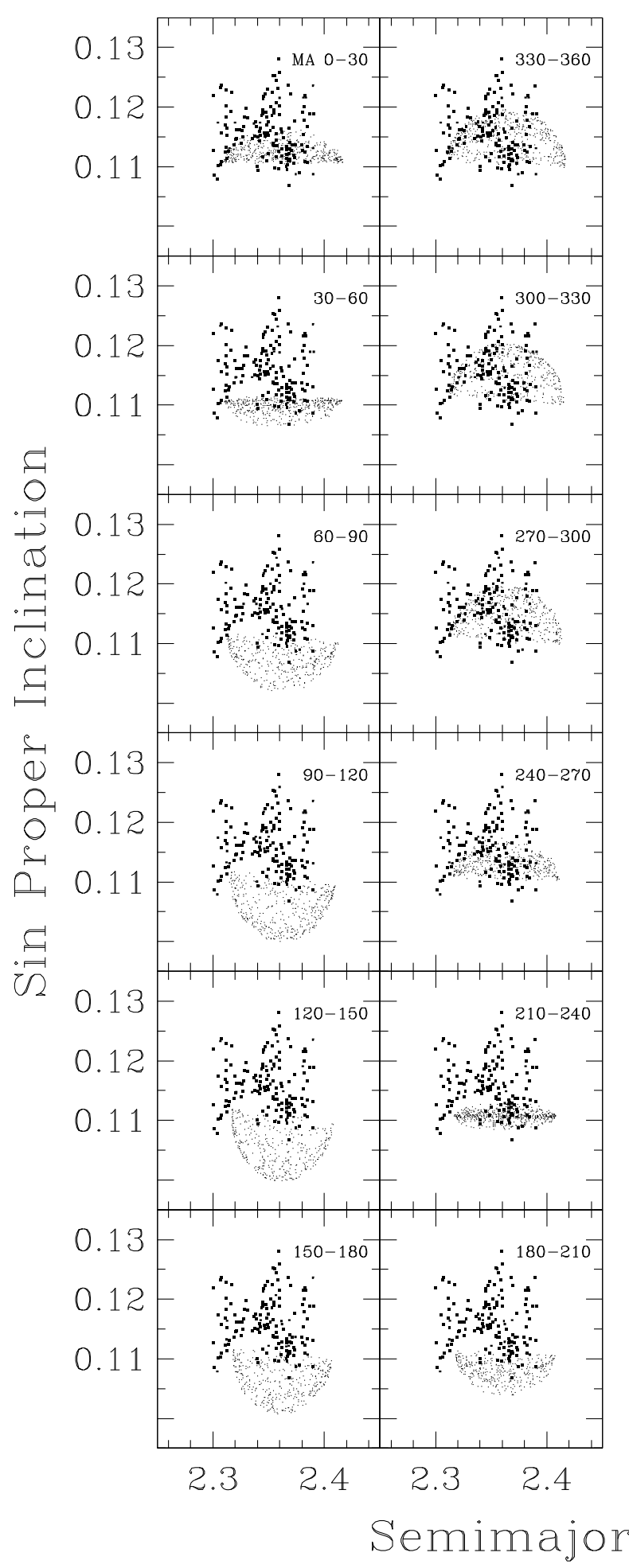

(b)

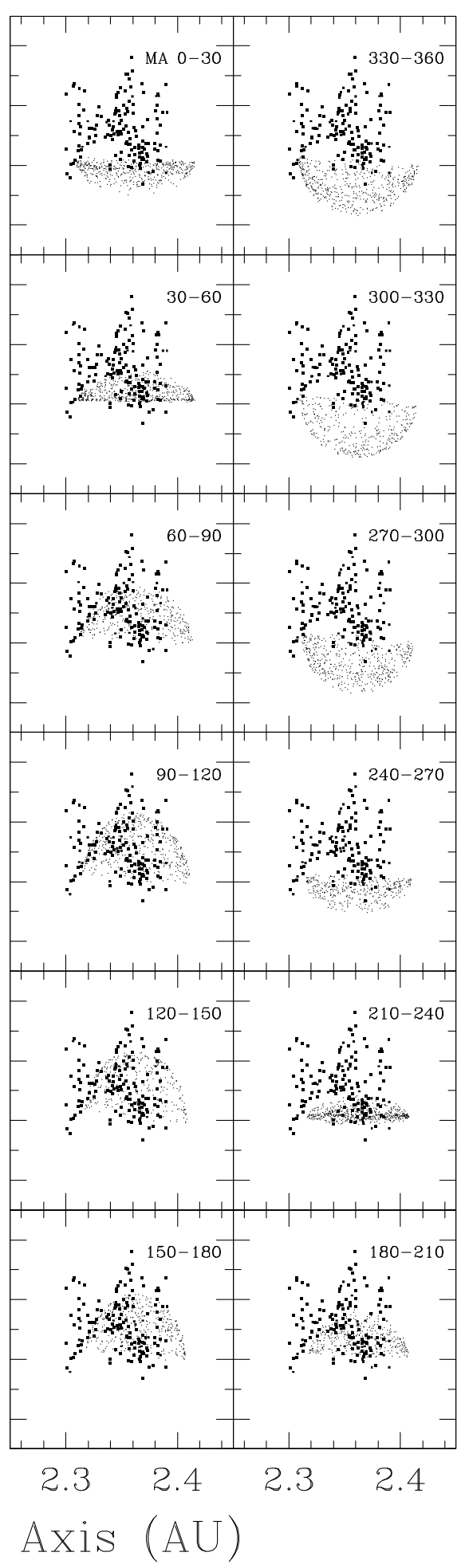

Fig. 2. Orbital inclinations of particles isotropically ejected from Vesta's (a) Northern and (b) Southern hemisphere are compared to those of Class 3 Vesta dynamical family members. In each frame, ejection occurs over 30 degrees of mean anomaly, increasing from 0-30 degrees in the upper left to $150-180$ degrees lower left and 330-360 degrees in the upper right.

$\mathrm{m} / \mathrm{s}$ would correspond to escape velocities of $440,560,720$, and $890 \mathrm{~m} / \mathrm{s}$, respectively.

Ejecting objects kilometers in diameter at such high speeds has been difficult to model, scaling from labora- tory impact experiments that predict much lower speeds (cf., Fujiwara et al., 1989). Hydrocode simulation of crater formation on Vesta by Ryan and Melosh (1998) did not produce ejecta speeds for km-sized objects that were high enough for 


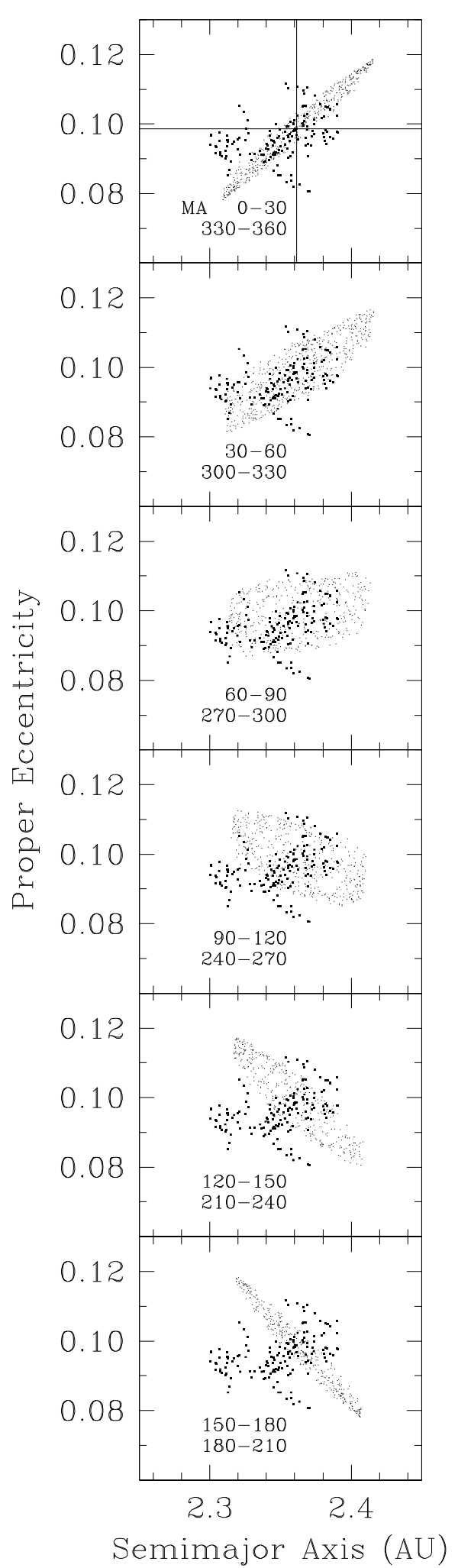

Fig. 3. Orbital eccentricities of particles isotropically ejected from Vesta's Northern and Southern hemisphere are compared to those of Class 3 Vesta dynamical family members. Because of Vesta's low inclination, Nothern and Southern hemispheric ejection produce nearly identical distributions at the scales shown. There is also mirroring over ranges of mean anomaly between the outbound and inbound legs of Vesta's orbit. In each frame, ejection occurs over 30 degrees of mean anomaly. escape. Using similar code, however, Asphaug (1997) has managed to simulate the event forming the $460-\mathrm{km}$ crater on Vesta and eject km-sized fragments at speeds of $600 \mathrm{~m} / \mathrm{s}$ without destroying Vesta's basaltic crust. These two results have yet to be resolved. Velocity diffusion arising from multiple asteroid collisions offers another possible explanation (cf., Marzari et al., 1995). This diffusion is produced by generations of collisional disruptions, over which smaller pieces of a once larger object tend to have increasing ranges of velocities relative to that of the original parent body (e.g., Vesta).

An alternative explanation for the origin of the more dynamically distinct Vestoids is that they do not originate from Vesta, but are the remaining fragments of a disrupted parent body geochemically similar to Vesta. Such an object would have disrupted a very long time ago, given that it had to be large enough to experience differentiation and given that the asteroid family its disruption would have created has largely eroded into the background population of asteroids.

\section{A Spectroscopic Clue}

Cochran and Vilas (1998) identified in the spectrum of Vesta an absorption feature corresponding to a spin-forbidden $\mathrm{Fe}^{2+}$ in six-fold coordination located near $505 \mathrm{~nm}$ (Fig. 5). The spectral placement, shape and existence as a singlet or a doublet are highly indicative of the presence and amount of $\mathrm{Ca}$ in pyroxene. This feature is seen in all laboratory spectra of HED meteorites (e.g., Gaffey, 1976; Hiroi et al., 1994) except for glassy diogenites (Vilas et al., 2000). Cochran and Vilas attribute this feature to augite (Ca-rich pyroxene), present as one component of HED meteorite pyroxenes. They further obtained complete longitudinal coverage of Vesta around $0^{\circ}$ latitude, observing this singlet feature in Vesta's spectrum, centered at $506.5 \mathrm{~nm}$, with a spectral width spanning 501.4 to $514.8 \mathrm{~nm}$. A drop in equivalent width of this feature corresponded to the topographic high located from $\sim 315^{\circ}$ to $0^{\circ}$ longitude, $-30^{\circ}$ latitude. This topographic high is interpreted to be material excavated and overturned as a result of the impact event that created the $460-\mathrm{km}$ crater near Vesta's south pole. This suggests that material interior to Vesta's surface is either augite-depleted or has a composition which otherwise masks the feature.

Vilas et al. (2000) studied the 505-nm feature in 13 asteroids dynamically linked to Vesta, or located between Vesta and the 3:1 resonance gap. Of these, two (1646 Rosseland, a C-type, and 2024 McLaughlin, an S-type) are "interlopers," seven are Class 3 Vesta dynamical family members (1933 Tichen, 3155 Lee, 3657 Ermolova, 3968 Koptelov, 4038 Kristina, 4147 Lennon, and 4546 Franck), and the four remaining are not Class 3 dynamical family members but are Vestoids (2011 Veteraniya, 2113 Ehrdni, 2442 Corbett, and 3869 Norton). Of the dynamical family members observed, one exhibited the 505-nm feature, one had a marginal detection, and five did not exhibit the feature. Of the four remaining Vestoids, all exhibited the feature, one marginally. These results are illustrated in Fig. 6.

The observations of Vilas et al. support the hypothesis that the Class 3 Vesta dynamical family members originate from a single impact forming the 460-km Southern hemisphere crater. In the course of that event, the volume of ma- 


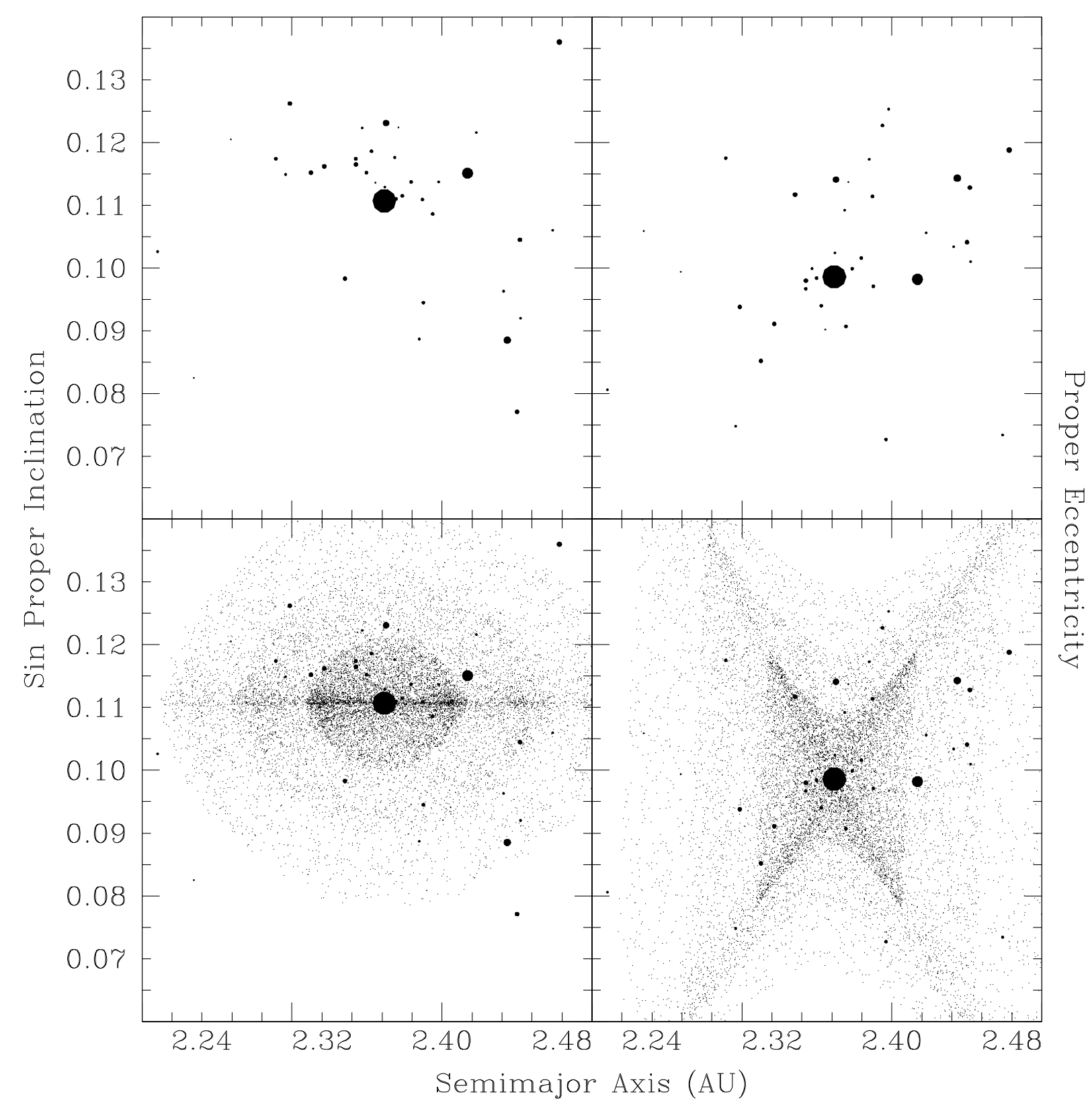

Fig. 4. Proper orbital element distribution of Vesta (large filled circle) and Vestoids (smaller filled circles) in the top panels, compared to the element distribution of test particles ejected (assuming zero escape velocity) isotropically over Vesta's orbit at 200 (inner zone), 400 (middle zone), and $600 \mathrm{~m} / \mathrm{s}$ (outer zone) in the lower panels.

terial ejected would have been interior to Vesta's surface. If Vesta's surface is covered by only a thin layer of augitecontaining pyroxene, then the dynamical family members should be dominated by the relatively augite-poor interior material. The more dynamically distinct Vestoids would have derived from some other collisional event preferentially excavating surface layers of Vesta (perhaps an highly oblique impact?), ejecting them at relatively high speeds (e.g., Yamamoto and Nakamura, 1998), or alternatively they derived from the disruption of a Vesta-like object that was more rich throughout with augite.

\section{The Origin of HED Meteorites}

HED meteorites appear to be more closely associated with the dynamically distinct Vestoids that share their 505-

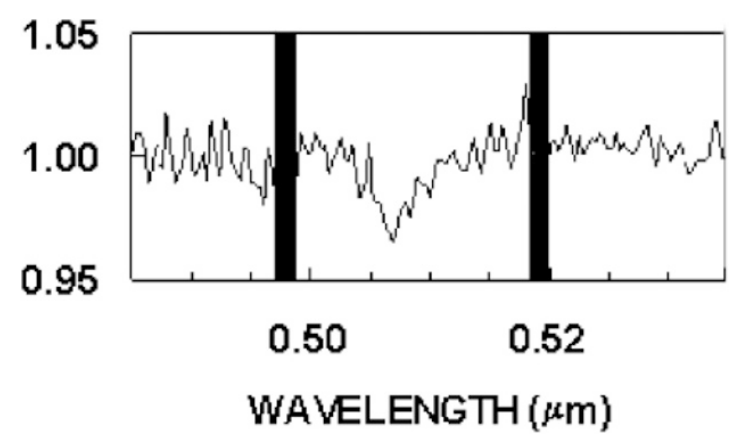

Fig. 5. The $505 \mathrm{~nm}$ feature, associated with augite, in an average of Vesta's solar-subtracted spectrum. From Jarvis et al. (2001). 


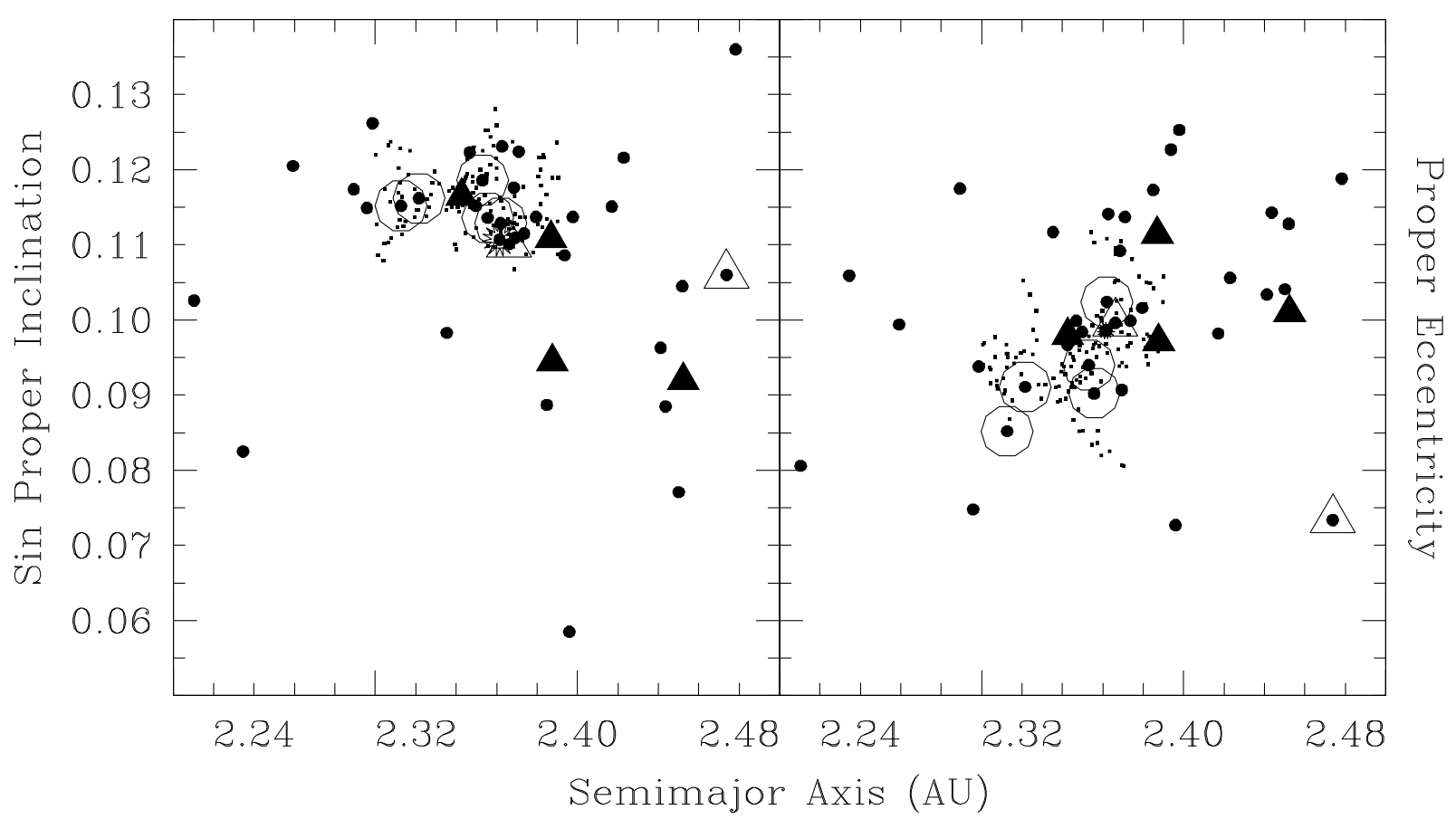

Fig. 6. Proper orbital elements of Class 3 Vesta family members (small dots) and Vestoids (large filled circles). Vesta is designated as a starburst. Large filled triangles indicate asteroids for which the 505-nm feature is strongly detected, open triangles denote marginal detection, and open circles denote no detection.

nm feature. Under this scenario, HED meteorites would not have originated from the collisional event that created the 460-km crater and Class 3 dynamical Vesta family members. Yet, the diogenites sample the deeper crust and mantle (cf., Consolmagno and Drake, 1977; Gaffey and McCord, 1978) and should be well represented spectroscopically in the Class 3 Vesta dynamical family members if they are associated with that crater-forming event. Thus, the HED meteorites may not be providing information about Vesta, but rather a similar parent body long ago destroyed. To better determine whether this is or is not the case, spectroscopic observations of many more bodies in this region (both dynamical family members and potential Vestoids) are needed.

\section{Conclusion}

The evolution of the association of HED meteorites with Vesta have grown from a spectroscopic correlation (cf., Consolmagno and Drake, 1977; Drake, 1979) to an overarching viewpoint which presumes that all basaltic asteroids, HED meteorites, and the impact forming the southern crater on Vesta are related (e.g., Binzel, 2001). The tension between this view and the existence of a basaltic asteroid beyond the 3:1 Kirkwood gap (Lazzaro et al., 2000), spectroscopic discrepancies among Vesta and many "Vestoids" (cf., Burbine and Binzel, 1997; Bell, 1998), and the present work point out the need for yet more observations and modeling. These may lead to a better understanding of how, in better detail, all of these objects are related to Vesta, or they may strengthen alternative hypotheses.

Acknowledgments. We would like to thank D. Britt and R. Binzel for their referee reports. MVS was supported by NASA Planetary Astronomy Grant NAG5-7758. FV also thanks the NASA Plane- tary Astronomy Program for its support.

\section{References}

Asphaug, E., Impact origin of the Vesta family, Meteor. and Planet. Sci., 32, 965-980, 1997.

Bell, J. F., The Vesta family: What went wrong, Bull. Am. Astr. Soc., 30, 504, 1998.

Binzel, R. P., Editorial: Forging the fourth link between planetary worlds: Vesta and the HEDs, Meteor. and Planet. Sci., 36, 479-480, 2001.

Binzel, R. P. and S. Xu, Chips off of asteroid 4 Vesta: Evidence for the parent body of basaltic achondrite meteorites, Science, 260, 186-191, 1993.

Burbine, T. H. and R. P. Binzel, SMASSIR measurements of Vesta chips: Evidence for weathering?, Bull. Am. Astr. Soc., 29, 964, 1997.

Bus, S. J., Compositional Structure in the Asteroid Belt: Results of a Spectroscopic Survey, Ph.D. Thesis, MIT, 1999.

Cochran, A. L. and F. Vilas, The changing spectrum of Vesta: Rotationally resolved spectroscopy of pyroxene on the surface, Icarus, 127, 121-129, 1998.

Consolmagno, G. J. and M. J. Drake, Composition and evolution of the eucrite parent body: Evidence from rare earth elements, Geochim. Cosmochim. Acta, 41, 1271-1282, 1977.

Drake, M., Geochemical evolution of the eucrite parent body-Possible nature and evolution of asteroid 4 Vesta, in Asteroids, edited by T. Gehrels, pp. 765-782, Univ. Arizona Press, Tucson, 1979.

Fujiwara, A., P. Cerroni, D. Davis, E. Ryan, M. Di Martino, K. Holsapple, and K. Housen, Experiments and scaling laws for catastrophic collisions, in Asteroids II, edited by R. Binzel, T. Gehrels, and M. Matthews, pp. 240-265, Univ. Arizona Press, Tucson, 1989.

Gaffey, M. J., Spectral reflectance properties of the meteorite classes, $J$. Geophys. Res., 81, 905-920, 1976.

Gaffey, M. J., Surface lithologic heterogeneity of Asteroid 4 Vesta, Icarus, 127, 130-157, 1997.

Gaffey, M. J. and T. B. McCord, Asteroid surface materials: Minerological characterizations from reflectance spectra, Space Sci. Rev., 21, 555-628, 1978.

Hilton, J. L., New masses and densities for 1 Ceres, 2 Pallas, and 4 Vesta, Bull. Am. Astr. Soc., 29, 1318, 1997.

Hirayama, K., Groups of asteroids probably of common origin, Astron. J., 31, 185-188, 1918.

Hiroi, T., C. M. Pieters, and H. Takeda, Grain size of the surface regolith of 
asteroid 4 Vesta estimated from its reflectance spectrum in comparison with HED meteorites, Meteoritics, 29, 394-396, 1994.

Jarvis, K. S., F. Vilas, A. L. Cochran, and M. S. Kelley, New visible-region spectral observations of Vesta and five Vestoids, LPSC XXXII, 2141, 2001.

Knežević, Z. and A. Milani, Synthetic proper elements for outer main belt asteroids, Celest. Mech. and Dyn. Astron., 78, 17-46, 2000.

Lazzaro, D., T. Michtchenko, J. M. Carvano, R. P. Binzel, S. J. Bus, T. H. Burbine, T. Mothe-Dinz, M. Florczak, C. A. Angeli, and A. W. Harris, Discovery of a basaltic asteroid in the outer main belt, Science, 288, 2033-2035, 2000

Marzari, F., D. Davis, and V. Vanzani, Collisional evolution of asteroid families, Icarus, 113, 168-187, 1995.

Marzari, F., A. Cellino, D. R. Davis, P. Farinella, V. Zappala, and V. Vanzani, Origin and evolution of the Vesta asteroid family, Astron. Astrophys., 316, 248-262, 1996.

McSween, H. A., Meteorites, in The New Solar System, edited by K. Beatty, C. Petersen, and A. Chaikin, pp. 351-364, Sky Publishing Corp., 1999.

Ryan, E. V. and H. J. Melosh, Impact fragmentation: From the laboratory to asteroids, Icarus, 133, 1-24, 1998.

Thomas, P. C., R. P. Binzel, M. J. Gaffey, B. H. Zellner, A. D. Storrs, and E. Wells, Vesta: Spin pole, size, and shape from HST images, Icarus, 128, 88-94, 1997.

Vilas, F., A. L. Cochran, and K. S. Jarvis, Vesta and the Vestoids: A new rock group?, Icarus, 147, 119-128, 2000.

Wisdom, J., Chaotic dynamics in the solar system, Icarus, 72, 241-275, 1987.

Wetherill, G. W., Where do the Apollo objects come from?, Icarus, 76, $1-18,1988$.

Yamamoto, S. and A. Nakamura, Velocity measurements of impact eject from regolith targets, Icarus, 128, 160-170, 1998.

Zappala, V., Ph. Bendjoya, A. Cellino, P. Farinella, and C. Froeschle, Asteroid families: Search of a 12,487-asteroid sample using two different clustering techniques, Icarus, 116, 291-314, 1995.

M. V. Sykes (e-mail: sykes@as.arizona.edu) and F. Vilas (e-mail: Faith.Vilas@jsc.nasa.gov) 